\title{
Generating varieties for the triple loop space of classical Lie groups
}

\author{
by \\ Yasuhiko Kamiyama (Okinawa)
}

\begin{abstract}
For $G=S U(n), S p(n)$ or $\operatorname{Spin}(n)$, let $C_{G}(S U(2))$ be the centralizer of a certain $S U(2)$ in $G$. We have a natural map $J: G / C_{G}(S U(2)) \rightarrow \Omega_{0}^{3} G$. For a generator $\alpha$ of $H_{*}\left(G / C_{G}(S U(2)) ; \mathbb{Z} / 2\right)$, we describe $J_{*}(\alpha)$. In particular, it is proved that $J_{*}$ : $H_{*}\left(G / C_{G}(S U(2)) ; \mathbb{Z} / 2\right) \rightarrow H_{*}\left(\Omega_{0}^{3} G ; \mathbb{Z} / 2\right)$ is injective.
\end{abstract}

1. Introduction. The purpose of this paper is to study an analogue of Bott's theorem on generating varieties when $S^{1}$ is replaced by $S U(2)$ and $\Omega G$ is replaced by $\Omega_{0}^{3} G$, where $G$ is a classical Lie group. We will explain the motivation for the study later. We recall Bott's theorem. Let $G$ be a compact simply connected Lie group. For a circle $S^{1}$ on $G$, let $C_{G}\left(S^{1}\right)$ be the centralizer of $S^{1}$ in $G$. We associate with it a map $f: G / C_{G}\left(S^{1}\right) \rightarrow \Omega G$ defined by $f\left(g C_{G}\left(S^{1}\right)\right)(x)=g x g^{-1} x^{-1}$, where $x \in S^{1}$. According to Bott [2], if $S^{1}$ is a suitable circle (more precisely, $S^{1}$ determines an element of $H_{1}(T ; \mathbb{Z})$, where $T$ is a maximal torus of $G$ containing $S^{1}$, and if the element is dual to a long root), then ( $S^{1}$ becomes a generating circle and) $f$ has the property that the image of $f_{*}: H_{*}\left(G / C_{G}\left(S^{1}\right) ; \mathbb{Z}\right) \rightarrow H_{*}(\Omega G ; \mathbb{Z})$ generates the Pontryagin ring $H_{*}(\Omega G ; \mathbb{Z})$. We call $G / C_{G}\left(S^{1}\right)$ a generating variety.

Let $S U(2)$ be a subgroup of $G$ and $C_{G}(S U(2))$ its centralizer in $G$. Hereafter we abbreviate $C_{G}(S U(2))$ to $C$ or $C_{G}$ and consider homology with $\mathbb{Z} / 2$-coefficients. We have a map

$$
J: G / C \rightarrow \Omega_{0}^{3} G
$$

defined by

$$
J(g C)(x)=g x g^{-1} x^{-1},
$$

where $x \in S U(2)$. For $G=S U(2), J$ is essentially the well known $J$-homomorphism $J: S O(3) \rightarrow \Omega_{1}^{3} S^{3}$. At present, the Pontryagin $\operatorname{ring} H_{*}\left(\Omega_{0}^{3} G ; \mathbb{Z} / 2\right)$

2000 Mathematics Subject Classification: Primary 58D27; Secondary 53C07, 55R40.

Key words and phrases: generating variety, instantons, triple loop space. 
is known for all $G$. (See [3] for $G=S U(n)$, [4] for $G=\operatorname{Spin}(n)$, [5] for $G=S p(n)$ and [6] for exceptional Lie groups.) The ring is polynomial with infinitely many generators and the generators are constructed as follows. From a finite number of elements $b_{\mu_{1}}, \ldots, b_{\mu_{r}}$ of $H_{*}\left(\Omega_{0}^{3} G ; \mathbb{Z} / 2\right)$, all the generators are constructed by applying the homology operations $Q_{1}, Q_{2}$ (and possibly $Q_{3}$ ). In this paper the subscript of an element means its degree, that is, $\operatorname{deg}\left(b_{\mu}\right)=\mu$. We call these $b_{\mu}$ fundamental generators. Now we consider the following questions:

(1) Is $J_{*}: H_{*}(G / C ; \mathbb{Z} / 2) \rightarrow H_{*}\left(\Omega_{0}^{3} G ; \mathbb{Z} / 2\right)$ injective?

(2) Does the image of $J_{*}$ contain all the fundamental generators?

The motivation for the questions is as follows. For a compact simple simply connected Lie group $G$, let $M(k, G)$ be the moduli space of based gauge equivalence classes of $G$-instantons over $S^{4}$ with instanton number $k$. Let $i_{k}: M(k, G) \rightarrow \Omega_{k}^{3} G$ be the inclusion. In [3] Boyer, Mann and Waggoner constructed non-trivial classes in $H_{*}(M(k, S U(n)) ; \mathbb{Z} / 2)$. For that purpose, they used a description of $M(1, G)$ in terms of a homogeneous space. More precisely, let $C=C_{G}(S U(2))$ be the centralizer of a certain $S U(2)$ in $G$. Then a result of [3] tells us that there exists a diffeomorphism $M(1, G) \cong$ $\mathbb{R}^{5} \times G / C$ such that the following diagram is homotopy commutative:

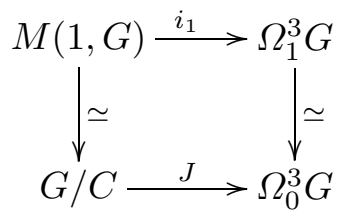

A crucial result of [3] determines $J_{*}(\alpha)$, where $\alpha \in H_{*}(S U(n) / C ; \mathbb{Z} / 2)$ is an even-dimensional generator (see Theorem 2.6). The motivation of this paper is to continue the study for $G=S U(n), S p(n)$, and $\operatorname{Spin}(n)(n \neq 4)$. Hence hereafter we consider an embedding of $S U(2)$ into $G$ so that $M(1, G) \cong$ $\mathbb{R}^{5} \times G / C$. (See [3], [9] or Sections 2-3.)

For $G / C$, we have the following examples:

EXAmple 1.1 ([3], [9]). (i) $S U(n) / C_{S U(n)}$ is diffeomorphic to the unit tangent bundle of $\mathbb{C} P^{n-1}$.

(ii) $S p(n) / C_{S p(n)}$ is diffeomorphic to $\mathbb{R} P^{4 n-1}$.

(iii) Since $\operatorname{Spin}(5) \cong S p(2)$ and $\operatorname{Spin}(6) \cong S U(4)$, we obtain examples of $\operatorname{Spin}(n) / C_{\operatorname{Spin}(n)}$ for $n=5$ and 6 from (i) and (ii).

In the main theorems of this paper, we describe $J_{*}(\alpha)$ for $\alpha$ a generator of $H_{*}(G / C ; \mathbb{Z} / 2)$. (See Theorem 2.7 for $G=S U(n)$ and $\alpha$ an odd-dimensional generator, Theorem 2.8 for $G=S p(n)$, and Theorem 3.4 for $G=\operatorname{Spin}(n)$.) In particular, we have the following: 
Theorem A. Let $G$ be $S U(n), S p(n)$, or $\operatorname{Spin}(n)(n \neq 4)$. Embed $S U(2)$ into $G$ so that $M(1, G) \cong \mathbb{R}^{5} \times G / C$. Then

(i) $J_{*}: H_{*}(G / C ; \mathbb{Z} / 2) \rightarrow H_{*}\left(\Omega_{0}^{3} G ; \mathbb{Z} / 2\right)$ is injective.

(ii) Excluding a fundamental generator in $H_{2 n-6}\left(\Omega_{0}^{3} \operatorname{Spin}(n) ; \mathbb{Z} / 2\right)$ for $n \equiv 3(\bmod 4)$, there are choices of the fundamental generators $b_{\mu}$ of $H_{*}\left(\Omega_{0}^{3} G ; \mathbb{Z} / 2\right)$ such that every $b_{\mu}$ is in the image of $J_{*}$.

If $\alpha \in H_{*}\left(G / C_{G} ; \mathbb{Z} / 2\right)$ is a stable element, that is, $\alpha$ is non-trivial in $H_{*}\left(G(\infty) / C_{G(\infty)} ; \mathbb{Z} / 2\right.$ ) (where $G(\infty)$ denotes $S U, S p$ or Spin), then we can use the Bott periodicity to study $J_{*}(\alpha)$ in $H_{*}\left(\Omega_{0}^{3} G ; \mathbb{Z} / 2\right.$ ) (see (5.4)). On the other hand, for $G=S U(n)$ or $\operatorname{Spin}(n), H_{*}\left(G / C_{G} ; \mathbb{Z} / 2\right)$ contains a nonstable element. The following theorem is a key to determining $J_{*}(\alpha)$ for $\alpha$ non-stable. For a map $f: X \rightarrow \Omega_{0}^{3} G$, let $\operatorname{Ad}(f): \Sigma^{3} X \rightarrow G$ be the adjoint map of $f$.

Theorem B. (i) For $G=S U(n)$ with $n$ even, let $i: S^{2 n-3} \rightarrow S U(n) / C$ be the inclusion of the fiber of the unit tangent bundle of $\mathbb{C} P^{n-1}$. Then $\operatorname{Ad}(J \circ i)$ generates the 2 -component of $\pi_{2 n}(S U(n)) \cong \mathbb{Z} / n$ !.

(ii) For $G=\operatorname{Spin}(n)$ with $n \neq 7$, there exists a spherical class in $H_{n-4}(\operatorname{Spin}(n) / C ; \mathbb{Z})$ which is denoted by $\nu: S^{n-4} \rightarrow \operatorname{Spin}(n) / C$. Then $\operatorname{Ad}(J \circ \nu)$ is a generator of $\pi_{n-1}(\operatorname{Spin}(n))$. (More precisely; $\operatorname{Ad}(J \circ \nu)$ is one of the generators for $n \equiv 0,1,2,4(\bmod 8) ; \operatorname{Ad}(J \circ \nu)$ has infinite order for $n$ even and is of order 2 for $n$ odd with $n \neq 7$.)

This paper is organized as follows. In Section 2 we study the cases $G=$ $S U(n)$ and $S p(n)$. Theorems 2.7 and 2.8 are the main results for $G=S U(n)$ and $G=S p(n)$ respectively. In Section 3 we study the case $G=\operatorname{Spin}(n)$, where Theorem 3.4 is the main result. Theorems 2.7 and 2.8 are proved in Section 4 and Theorem 3.4 is proved in Section 5, where Theorem B is a key for the proofs.

2. Main results for $G=S U(n)$ and $S p(n)$. First we study the case $G=S U(n)$. Since $S U(2)=S p(1)$, we assume $n \geq 3$. (The (2.2) below holds for $n=2$, but it is more natural to consider (2.4) for $n \geq 3$.) We embed $S U(2)$ into $S U(n)$ as the first $2 \times 2$ elements. Then $C=C_{S U(n)}$ is given by the following set of matrices:

$$
C=\left\{\left(\begin{array}{lll}
a & 0 & 0 \\
0 & a & 0 \\
0 & 0 & A
\end{array}\right): A \in U(n-2), a^{2} \operatorname{det} A=1\right\} .
$$

Therefore $S U(n) / C$ is diffeomorphic to the unit tangent bundle of $\mathbb{C} P^{n-1}$ (see [3], [9] or (4.1)) and $H^{*}(S U(n) / C ; \mathbb{Z} / 2)$ is given as follows: 
(1) For $n$ even,

$$
H^{*}(S U(n) / C ; \mathbb{Z} / 2) \cong H^{*}\left(\mathbb{C} P^{n-1} ; \mathbb{Z} / 2\right) \otimes H^{*}\left(S^{2 n-3} ; \mathbb{Z} / 2\right) .
$$

(2) For $n$ odd,

$$
H^{*}(S U(n) / C ; \mathbb{Z} / 2) \cong H^{*}\left(\mathbb{C} P^{n-2} ; \mathbb{Z} / 2\right) \otimes H^{*}\left(S^{2 n-1} ; \mathbb{Z} / 2\right) .
$$

We write the generators of $H_{*}(S U(n) / C ; \mathbb{Z} / 2)$ as follows.

(1) For $n$ even,

$$
\begin{cases}\alpha_{2 i}, & 1 \leq i \leq n-1 \\ \beta_{2 i+1}, & n-2 \leq i \leq 2 n-3\end{cases}
$$

(2) For $n$ odd,

$$
\begin{cases}\alpha_{2 i}, & 1 \leq i \leq n-2, \\ \beta_{2 i+1}, & n-1 \leq i \leq 2 n-3 .\end{cases}
$$

The structure of $H_{*}\left(\Omega_{0}^{3} S U(n) ; \mathbb{Z} / 2\right)$ is given in [3]. First, the following elements are defined in $H_{*}\left(\Omega_{0}^{3} S U(n) ; \mathbb{Z} / 2\right)$ :

$$
\begin{cases}x_{2}, & \\ y_{2 i}, & 2 \leq i \leq n-2, i \equiv 0(\bmod 2), \\ z_{4 i+1}, & {\left[\frac{n-1}{2}\right] \leq i \leq n-2, i \equiv 1(\bmod 2) .}\end{cases}
$$

Here $x_{2}$ is defined by $x_{2}=Q_{2}[1] *[-2]$. (2.4) gives the set of fundamental generators of $H_{*}\left(\Omega_{0}^{3} S U(n) ; \mathbb{Z} / 2\right)$. The set of all ring generators is as follows:

$$
\begin{cases}Q_{2}^{a}\left(x_{2}\right), & a \geq 0, \\ Q_{2}^{a}\left(y_{2 i}\right), & a \geq 0,2 \leq i \leq\left[\frac{n-3}{2}\right], i \equiv 0(\bmod 2), \\ Q_{1}^{a} Q_{2}^{b}\left(y_{2 i}\right), & a, b \geq 0,\left[\frac{n-1}{2}\right] \leq i \leq n-2, i \equiv 0(\bmod 2), \\ Q_{1}^{a} Q_{3}^{b}\left(z_{4 i+1}\right), & a, b \geq 0,\left[\frac{n-1}{2}\right] \leq i \leq n-2, i \equiv 1(\bmod 2) .\end{cases}
$$

Consider the following diagram:

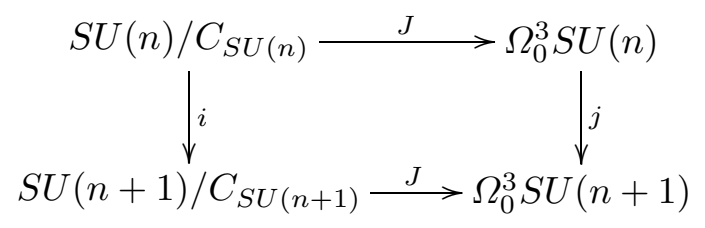

where $i$ and $j$ are the inclusions. If $\alpha_{2 i}$ is defined both in $H_{2 i}\left(S U(n) / C_{S U(n)}\right.$; $\mathbb{Z} / 2)$ and $H_{2 i}\left(S U(n+1) / C_{S U(n+1)} ; \mathbb{Z} / 2\right)$, then $\alpha_{2 i}$ in the top row is mapped by $i_{*}$ to $\alpha_{2 i}$ in the bottom row. Similar remarks hold for $\beta_{2 i+1}$ with respect to $i_{*}$ and for $x_{2}, y_{2 i}$ and $z_{4 i+1}$ with respect to $j_{*}$.

$J_{*}\left(\alpha_{2 i}\right)$ is known:

TheOREM 2.6 ([3]). There are choices of the fundamental generators $y_{2 i}$ such that: 
(i) For $i$ even, $J_{*}\left(\alpha_{2 i}\right)=y_{2 i}$.

(ii) For $i$ odd, $J_{*}\left(\alpha_{2 i}\right)$ contains the term $x_{2} * y_{2 i-2}$.

Our main result for $G=S U(n)$ is as follows.

THEOREM 2.7. There are choices of the fundamental generators $z_{4 i+1}$ (where $i \equiv 1(\bmod 2))$ such that

(i) $J_{*}\left(\beta_{8 k+1}\right)$ contains the term $Q_{1}\left(y_{4 k}\right)$.

(ii) $J_{*}\left(\beta_{8 k+3}\right)$ contains the term $x_{2} * Q_{1}\left(y_{4 k}\right)$.

(iii) $J_{*}\left(\beta_{8 k+5}\right)=z_{8 k+5}$.

(iv) $J_{*}\left(\beta_{8 k+7}\right)$ contains the term $x_{2} * z_{8 k+5}$.

REMARK. In [3], $J_{*}\left(\beta_{5}\right)$ is also studied.

From Theorems 2.6, 2.7 and the structure of $H^{*}(S U(n) / C ; \mathbb{Z} / 2)$, we see that $J^{*}: H^{*}\left(\Omega_{0}^{3} S U(n) ; \mathbb{Z} / 2\right) \rightarrow H^{*}(S U(n) / C ; \mathbb{Z} / 2)$ is surjective, hence Theorem A holds for $G=S U(n)$.

Next we study the case $G=S p(n)$. We embed $S p(1)$ into $S p(n)$ as the first $1 \times 1$ element. Then $C=C_{S p(n)}=\mathbb{Z} / 2 \times S p(n-1)$, hence $S p(n) / C$ is diffeomorphic to $\mathbb{R} P^{4 n-1}$. We write the generators of $H_{*}(S p(n) / C ; \mathbb{Z} / 2)$ as $\alpha_{\mu}(1 \leq \mu \leq 4 n-1)$. The structure of $H_{*}\left(\Omega_{0}^{3} S p(n) ; \mathbb{Z} / 2\right)$ is given in [5]. The result is

$$
\begin{aligned}
H_{*}\left(\Omega_{0}^{3} S p(n) ; \mathbb{Z} / 2\right) \cong & \mathbb{Z} / 2\left[Q_{1}^{a} Q_{2}^{b}[1] *\left[-2^{a+b}\right]: a, b \geq 0\right] \\
& \otimes \mathbb{Z} / 2\left[Q_{1}^{a} Q_{2}^{b}\left(z_{4 i}\right): a, b \geq 0,1 \leq i \leq n-1\right] .
\end{aligned}
$$

Setting $x_{1}=Q_{1}[1] *[-2]$ and $y_{2}=Q_{2}[1] *[-2]$, we have the set of fundamental generators $x_{1}, y_{2}$ and $z_{4 i}(1 \leq i \leq n-1)$.

Our result for $G=S p(n)$ is as follows.

THEOREM 2.8. There are choices of the fundamental generators $z_{4 i}$ such that

(i) $J_{*}\left(\alpha_{1}\right)=x_{1}, J_{*}\left(\alpha_{2}\right)=y_{2}$ and $J_{*}\left(\alpha_{4 i}\right)=z_{4 i}(1 \leq i \leq n-1)$.

(ii) In general, for $1 \leq \mu \leq 4 n-1$, set $\mu=\varepsilon_{1}+2 \varepsilon_{2}+4 i$, where $\varepsilon_{j}=0$ or 1 . Then $J_{*}\left(\alpha_{\mu}\right)$ contains the term $x_{1}^{\varepsilon_{1}} * y_{2}^{\varepsilon_{2}} * z_{4 i}$.

Theorem A for $G=S p(n)$ follows from Theorem 2.8 and the structure of $H^{*}(S p(n) / C ; \mathbb{Z} / 2)$.

We prove Theorems 2.7 and 2.8 in Section 4.

3. Main result for $G=\operatorname{Spin}(n)$. Since $\operatorname{Spin}(3) \cong S U(2)$ and $\operatorname{Spin}(4)$ is not simple, we assume $n \geq 5$. Recall that $\operatorname{Spin}(4) \cong S_{1}^{3} \times S_{2}^{3}$, where $S_{1}^{3}$ and $S_{2}^{3}$ are two copies of $\operatorname{Spin}(3) \cong S^{3}$. (In Section 5 we write $S_{i}^{3}$ in terms of the Clifford algebra.) We embed $\operatorname{Spin}(3)$ into $\operatorname{Spin}(n)$ as $S_{1}^{3}$. Then $C=$ $C_{\operatorname{Spin}(n)}=\operatorname{Spin}(n-4) \times S_{2}^{3}$. We recall the structure of $H^{*}(\operatorname{Spin}(n) / C ; \mathbb{Z} / 2)$. 
Proposition 3.1 ([7]). Let $n=4 t+l$ with $0 \leq l \leq 3$. Then we have the following isomorphism of modules:

$$
H^{*}(\operatorname{Spin}(n) / C ; \mathbb{Z} / 2) \cong \mathbb{Z} / 2\left[c_{2}\right] /\left(c_{2}^{t}\right) \otimes \Delta\left(u_{i_{1}}, u_{i_{2}}, u_{i_{3}}\right),
$$

where $\operatorname{deg} c_{2}=4$ and $\operatorname{deg} u_{i_{j}}=i_{j}$ with

$$
\left(i_{1}, i_{2}, i_{3}\right)= \begin{cases}(4 t-4,4 t-3,4 t-2), & n=4 t \\ (4 t-3,4 t-2,4 t), & n=4 t+1, \\ (4 t-2,4 t, 4 t+1), & n=4 t+2, \\ (4 t, 4 t+1,4 t+2), & n=4 t+3 .\end{cases}
$$

Here $\Delta\left(x_{1}, \ldots, x_{m}\right)$ denotes the graded algebra over $\mathbb{Z} / 2$ with $\mathbb{Z} / 2$-basis $\left\{x_{i_{1}} \ldots x_{i_{r}}: 1 \leq i_{1}<\ldots<i_{r} \leq m\right\}$. (If we add the relations $x_{i}^{2}=0$, $\Delta\left(x_{1}, \ldots, x_{m}\right)$ becomes the exterior algebra $\Lambda\left(x_{1}, \ldots, x_{m}\right)$.)

The mod 2 cohomology ring with squaring operations is also determined in [7]. By Proposition 3.1, we can define elements of $H_{*}(\operatorname{Spin}(n) / C ; \mathbb{Z} / 2)$ as follows. Note that two of $u_{i_{j}}$ are even-dimensional and one of them has dimension congruent to $1 \bmod 4$. We write them as $u_{2 a_{j}}(j=1,2)$ and $u_{4 b+1}$. Hence $a_{2}=a_{1}+1$. We set

$$
\begin{cases}\alpha_{4 i}=\left(c_{2}^{i}\right)^{*}, & 1 \leq i \leq t-1 \\ \beta_{4 b+4 i+1}=\left(c_{2}^{i} u_{4 b+1}\right)^{*}, & 0 \leq i \leq t-1 \\ \gamma_{2 a_{j}+4 i}=\left(c_{2}^{i} u_{2 a_{j}}\right)^{*}, & 0 \leq i \leq t-1, j=1,2,\end{cases}
$$

where $(\cdot)^{*}$ denotes the dual element with respect to the monomial basis.

The structure of $H_{*}\left(\Omega_{0}^{3} \operatorname{Spin}(n) ; \mathbb{Z} / 2\right)$ is given in [4]. It depends on the congruence class of $n \bmod 8$. For example, for $n=8 g+2$, we have the following set of fundamental generators of $H_{*}\left(\Omega_{0}^{3} \operatorname{Spin}(8 g+2) ; \mathbb{Z} / 2\right)$ :

$$
\begin{cases}x_{4 k}, & 1 \leq k \leq 2 g-1, \\ y_{8 g+8 k+5}, & 0 \leq k \leq g-1, \\ z_{8 g+2 k}, & -1 \leq k \leq 4 g-2, k \neq 1(\bmod 4) .\end{cases}
$$

Hence $y_{\mu}$ is defined only when $\mu \equiv 5(\bmod 8)$ and $z_{\mu}$ is defined only when $\mu \equiv 0,4$ or $6(\bmod 8)$. We define $y_{\mu}$ for $\mu \equiv 1(\bmod 8)$ and $z_{\mu}$ for $\mu \equiv 2$ $(\bmod 8)$ by setting

$$
\begin{cases}y_{8 g+8 k+1}=Q_{1}\left(x_{4 g+4 k}\right), & 0 \leq k \leq g-1 \\ z_{8 g+2 k}=Q_{2}\left(x_{4 g+k-1}\right), & 1 \leq k \leq 4 g-3, k \equiv 1(\bmod 4) .\end{cases}
$$

The elements of (3.3) are not fundamental generators. We do the same procedure for all $n$. Then we obtain the following elements of $H_{*}\left(\Omega_{0}^{3} \operatorname{Spin}(n) ; \mathbb{Z} / 2\right)$, among which the elements of the form (3.3) are not fundamental generators. 
(1) For $n=4 t$,

$$
\begin{cases}x_{4 i}, & 1 \leq i \leq t-1 \\ y_{4 t+4 i-3}, & 0 \leq i \leq t-1 \\ z_{4 t+2 i-4}, & 0 \leq i \leq 2 t-1\end{cases}
$$

(2) For $n=4 t+1$,

$$
\begin{cases}x_{4 i}, & 1 \leq i \leq t-1 \\ y_{4 t+4 i-3}, & 0 \leq i \leq t-1 \\ z_{4 t+2 i-2}, & 0 \leq i \leq 2 t-1 .\end{cases}
$$

(3) For $n=4 t+2$,

$$
\begin{cases}x_{4 i}, & 1 \leq i \leq t-1 \\ y_{4 t+4 i+1}, & 0 \leq i \leq t-1 \\ z_{4 t+2 i-2}, & 0 \leq i \leq 2 t-1\end{cases}
$$

(4) For $n=4 t+3$,

$$
\begin{cases}x_{4 i}, & 1 \leq i \leq t-1, \\ y_{4 t+4 i+1}, & 0 \leq i \leq t-1, \\ z_{4 t+2 i}, & 0 \leq i \leq 2 t .\end{cases}
$$

For $\alpha_{\mu}, \beta_{\mu}, \gamma_{\mu}$ in (3.2) and $x_{\mu}, y_{\mu}, z_{\mu}$, a similar naturality to (2.5) holds. Note that $\alpha_{\mu}, \beta_{\mu}, \gamma_{\mu}$ correspond to $x_{\mu}, y_{\mu}, z_{\mu}$ respectively except the fundamental generator $z_{8 t}$ in $H_{8 t}\left(\Omega_{0}^{3} \operatorname{Spin}(n) ; \mathbb{Z} / 2\right)$ for $n=4 t+3$. The following main theorem asserts that the correspondence is realized through $J_{*}$ :

TheOREM 3.4. There are choices of the fundamental generators $x_{\mu}$ for $\mu \equiv 0(\bmod 4), y_{\mu}$ for $\mu \equiv 5(\bmod 8)$ and $z_{\mu}$ for $\mu \equiv 0,4,6(\bmod 8)$ such that

(i) $J_{*}\left(\alpha_{\mu}\right)=x_{\mu}$.

(ii) (a) When $\mu \equiv 5(\bmod 8), J_{*}\left(\beta_{\mu}\right)=y_{\mu}$.

(b) When $\mu \equiv 1(\bmod 8), J_{*}\left(\beta_{\mu}\right)$ contains the term $y_{\mu}$.

(iii) (a) When $\mu \equiv 0,4$ or $6(\bmod 8), J_{*}\left(\gamma_{\mu}\right)=z_{\mu}$.

(b) When $\mu \equiv 2(\bmod 8), J_{*}\left(\gamma_{\mu}\right)$ contains the term $z_{\mu}$.

Note that $y_{\mu}$ in Theorem 3.4(ii)(b) and $z_{\mu}$ in (iii)(b) are not fundamental generators. (Compare (3.3).)

Theorem A for $G=\operatorname{Spin}(n)$ follows from Theorem 3.4 and the structure of $H^{*}(\operatorname{Spin}(n) / C ; \mathbb{Z} / 2)$ (see Proposition 3.1).

We prove Theorem 3.4 in Section 5. 
4. Proofs of Theorems 2.7 and 2.8. First we prove Theorem B(i). Recall that $C=C_{S U(n)}$ is given in (2.1). We set

$$
\begin{aligned}
S U(n-2) & =\left\{\left(\begin{array}{lll}
1 & 0 & 0 \\
0 & 1 & 0 \\
0 & 0 & A
\end{array}\right): A \in S U(n-2)\right\}, \\
S U(n-1) & =\left\{\left(\begin{array}{ll}
1 & 0 \\
0 & B
\end{array}\right): B \in S U(n-1)\right\}, \\
U(n-1) & =\left\{\left(\begin{array}{ll}
a & 0 \\
0 & B
\end{array}\right): B \in U(n-1), a \operatorname{det} B=1\right\} .
\end{aligned}
$$

We have the following fiber bundle which realizes $S U(n) / C$ as the unit tangent bundle of $\mathbb{C} P^{n-1}$ :

$$
S U(n-1) / S U(n-2) \stackrel{i}{\rightarrow} S U(n) / C \rightarrow S U(n) / U(n-1),
$$

where $i$ is the inclusion. We obtain a map $\operatorname{Ad}(J \circ i): S^{2 n-3} \wedge S^{3} \rightarrow S U(n)$. Consider the homotopy sequence of a principal bundle

$$
S U(n-1) \rightarrow S U(n) \stackrel{p}{\rightarrow} S^{2 n-1} .
$$

Since

$$
\pi_{2 n-1}(S U(n-1)) \cong \begin{cases}0, & n \text { even } \\ \mathbb{Z} / 2, & n \text { odd }\end{cases}
$$

(see, for example, [8]), $p_{*}: \pi_{2 n}(S U(n)) \rightarrow \pi_{2 n}\left(S^{2 n-1}\right)$ is surjective for $n$ even. Hence in order to prove Theorem B(i), it suffices to prove the following:

Proposition 4.2. For $n$ even, set $\phi=p \circ \operatorname{Ad}(J \circ i)$. Then $\phi$ is a generator of $\pi_{2 n}\left(S^{2 n-1}\right)$.

Proof. First we calculate $\phi$ explicitly. Let $\left(z_{1}, \ldots, z_{n-1}\right) \wedge(a, b) \in S^{2 n-3}$ $\wedge S^{3}$. We use the following notations.

(1) A matrix in $S U(n)$ which represents $\left(z_{1}, \ldots, z_{n-1}\right)$ is given by

$$
g=\left(\begin{array}{ccccc}
1 & 0 & 0 & \ldots & 0 \\
0 & z_{1} & 0 & \ldots & 0 \\
\vdots & \vdots & & \mathrm{A} & \\
0 & z_{n-1} & & &
\end{array}\right)
$$

where

$$
\left(\begin{array}{cccc}
z_{1} & 0 & \ldots & 0 \\
\vdots & & \mathrm{A} & \\
z_{n-1} & & &
\end{array}\right) \in S U(n-1)
$$


(2) A matrix in $S U(n)$ which corresponds to $(a, b)$ is given by

$$
x=\left(\begin{array}{crc}
a & -\bar{b} & 0 \\
b & \bar{a} & \\
& 0 & E_{n-2}
\end{array}\right)
$$

where $E_{n-2}$ denotes the unit matrix.

(3) We define the projection $p: S U(n) \rightarrow S^{2 n-1}$ by mapping an element of $S U(n)$ to its first row.

Now it is easy to calculate the first row of $g x g^{-1} x^{-1}$. The result is

$$
\phi\left(\left(z_{1}, \ldots, z_{n-1}\right) \wedge(a, b)\right)=\left(|b|^{2} \bar{z}_{1}+|a|^{2}, a \bar{b}\left(1-\bar{z}_{1}\right),-\bar{b} \bar{z}_{2}, \ldots,-\bar{b} \bar{z}_{n-1}\right) .
$$

Define $\psi: S^{2 n-3} \wedge S^{3} \rightarrow S^{2 n-1}$ by

$$
\psi\left(\left(z_{1}, \ldots, z_{n-1}\right) \wedge(a, b)\right)=\left(|b|^{2} z_{1}+|a|^{2}, b z_{2}, \ldots, b z_{n-1}, a b\left(1-z_{1}\right)\right) .
$$

It is clear that $\phi \simeq \psi$.

Next we calculate the generator of $\pi_{2 n}\left(S^{2 n-1}\right)$ explicitly. The following lemma is well known (see, for example, [11, p. 107]).

Lemma 4.4. The map $j: S^{m} \wedge S^{p} \rightarrow S^{m+p}$ defined by

$$
\begin{array}{r}
j\left(\left(u_{1}, \ldots, u_{m+1}\right) \wedge\left(x_{1}, \ldots, x_{p+1}\right)\right)=\left(\frac{\left(1-x_{1}\right) u_{1}+\left(1+x_{1}\right)}{2}, \frac{\left(1-x_{1}\right) u_{2}}{2},\right. \\
\left.\ldots, \frac{\left(1-x_{1}\right) u_{m+1}}{2}, \sqrt{\frac{1-u_{1}}{2}} x_{2}, \ldots, \sqrt{\frac{1-u_{1}}{2}} x_{p+1}\right)
\end{array}
$$

is a homeomorphism.

Let $\eta: S^{3} \rightarrow S^{2}$ be the Hopf map. It is given by $\eta(a, b)=\left(1-2|b|^{2}, 2 a b\right) \in$ $S^{2} \subset \mathbb{R} \times \mathbb{C}$. Then $\Sigma^{2 n-3} \eta$ is the generator of $\pi_{2 n}\left(S^{2 n-1}\right)$, and using Lemma 4.4, we have

$$
\begin{aligned}
& \Sigma^{2 n-3} \eta\left(\left(z_{1}, \ldots, z_{n-1}\right)\right.\wedge(a, b)) \\
&=\left(|b|^{2} z_{1}+|a|^{2},|b|^{2} z_{2}, \ldots,|b|^{2} z_{n-1}, a b \sqrt{2\left(1-\operatorname{Re} z_{1}\right)}\right) .
\end{aligned}
$$

Now we have two maps, $\psi$ in (4.3) and $\Sigma^{2 n-3} \eta$ in (4.5). We prove that $\psi \simeq \Sigma^{2 n-3} \eta$ when $n$ is even. We define a map $h: S^{2 n-1} \rightarrow S^{2 n-2}$ by

$$
h\left(w_{1}, \ldots, w_{n}\right)=\frac{\left(w_{1}, 2 \bar{w}_{2} w_{3},\left|w_{2}\right|^{2}-\left|w_{3}\right|^{2}, w_{4}, \ldots, w_{n}\right)}{\sqrt{\left|w_{1}\right|^{2}+\left(\left|w_{2}\right|^{2}+\left|w_{3}\right|^{2}\right)^{2}+\sum_{i=4}^{n}\left|w_{i}\right|^{2}}} .
$$

It is easy to see that $h \simeq \Sigma^{2 n-4} \eta$, hence the composition $h \circ: \pi_{2 n}\left(S^{2 n-1}\right) \rightarrow$ $\pi_{2 n}\left(S^{2 n-2}\right)$ is an isomorphism. We define a map $\psi_{1}: S^{2 n-3} \wedge S^{3} \rightarrow S^{2 n-1}$ by

$$
\begin{aligned}
\psi_{1}\left(\left(z_{1}, \ldots, z_{n-1}\right)\right. & \wedge(a, b)) \\
= & \left(|b|^{2} z_{1}+|a|^{2},|b| z_{2},|b| z_{3}, b z_{4}, \ldots, b z_{n-1}, a b\left(1-z_{1}\right)\right) .
\end{aligned}
$$


It is clear that $h \circ \psi=h \circ \psi_{1}$, hence $\psi \simeq \psi_{1}$. This process implies that two coordinates of $\psi$ can be changed simultaneously from $b z_{i}$ to $|b| z_{i}$. Since $n$ is even, we see that $\psi$ is homotopic to the map $\widetilde{\psi}: S^{2 n-3} \wedge S^{3} \rightarrow S^{2 n-1}$ defined by

$$
\widetilde{\psi}\left(\left(z_{1}, \ldots, z_{n-1}\right) \wedge(a, b)\right)=\left(|b|^{2} z_{1}+|a|^{2},|b| z_{2}, \ldots,|b| z_{n-1}, a b\left(1-z_{1}\right)\right) .
$$

Finally, using (4.5), it is easy to construct a homotopy $\widetilde{\psi} \simeq \Sigma^{2 n-3} \eta$. This completes the proof of Proposition 4.2, and hence also that of Theorem B(i).

For $n$ even, let $\sigma_{2 n-3} \in H_{2 n-3}\left(S^{2 n-3} ; \mathbb{Z} / 2\right)$ be the generator, where $S^{2 n-3}$ is the fiber of (4.1). By $(2.2)$, we have $i_{*}\left(\sigma_{2 n-3}\right)=\beta_{2 n-3}$. Recall that we have a map $J \circ i: S^{2 n-3} \rightarrow \Omega_{0}^{3} S U(n)$.

LeMma 4.6. $(J \circ i)_{*}\left(\sigma_{2 n-3}\right)$ is non-zero in $H_{2 n-3}\left(\Omega_{0}^{3} S U(n) ; \mathbb{Z} / 2\right)$.

Proof. Let $W$ be the homotopy-theoretic fiber of the inclusion $\Omega_{0}^{3} S U(n)$ $\hookrightarrow \Omega_{0}^{3} S U \simeq B U$. Consider the following diagram:

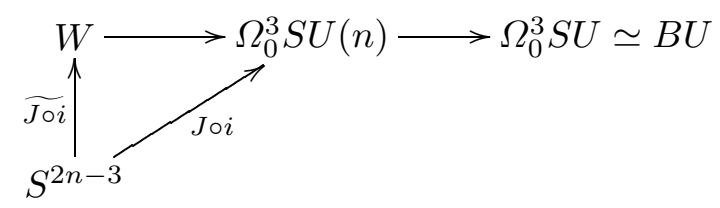

where $\widetilde{J \circ} i$ is the lift of $J \circ i$. The first non-vanishing homotopy of $W$ is $\pi_{2 n-3}(W) \cong \mathbb{Z}$ and $\widetilde{J \circ} i$ is not divisible by 2 by Theorem $\mathrm{B}(\mathrm{i})$. For $n$ even, $H_{2 n-3}(W ; \mathbb{Z} / 2) \rightarrow H_{2 n-3}\left(\Omega_{0}^{3} S U(n) ; \mathbb{Z} / 2\right)$ is an isomorphism by (2.4). Hence $(J \circ i)_{*}\left(\sigma_{2 n-3}\right) \neq 0$.

Proposition 4.7. In $H_{2 n-3}\left(\Omega_{0}^{3} S U(n) ; \mathbb{Z} / 2\right)$,

$$
J_{*}\left(\beta_{2 n-3}\right)= \begin{cases}Q_{1}\left(y_{4 k}\right), & n=4 k+2, \\ z_{8 k+5}, & n=4 k+4 .\end{cases}
$$

Proof. By Lemma 4.6, $J_{*}\left(\beta_{2 n-3}\right) \neq 0$ in $H_{2 n-3}\left(\Omega_{0}^{3} S U(n) ; \mathbb{Z} / 2\right)$. By $(2.4)$, $H_{2 n-3}\left(\Omega_{0}^{3} S U(n) ; \mathbb{Z} / 2\right) \cong \mathbb{Z} / 2$ and the generator is as on the right-hand side of the conclusion. Hence the result follows.

Proof of Theorem 2.7. If $\beta_{2 n-3}$ is defined in $H_{2 n-3}\left(S U(d) / C_{S U(d)} ; \mathbb{Z} / 2\right)$, then $d \leq n$. Using (2.5) we can regard Proposition 4.7 as relations in $H_{2 n-3}\left(S U(d) / C_{S U(d)} ; \mathbb{Z} / 2\right)$ for each $d$ for which $\beta_{2 n-3}$ is defined. For $\beta_{2 n-3}$ with $n=4 k+2$, the relation is up to the kernel of $i_{*}: H_{2 n-3}\left(\Omega_{0}^{3} S U(d) ; \mathbb{Z} / 2\right)$ $\rightarrow H_{2 n-3}\left(\Omega_{0}^{3} S U(n) ; \mathbb{Z} / 2\right)$, since $Q_{1}\left(y_{4 k}\right)$ is a fixed element. On the other hand, for $\beta_{2 n-3}$ with $n=4 k+4$, we can define $z_{8 k+5}$ to be $J_{*}\left(\beta_{2 n-3}\right)$. Therefore (i) and (iii) of Theorem 2.7 hold.

Consider $\beta_{2 n-1} \in H_{2 n-1}(S U(n) / C ; \mathbb{Z} / 2)$ for $n$ even. Since $\Delta_{*}\left(\beta_{2 n-1}\right)$ contains the term $\alpha_{2} \otimes \beta_{2 n-3}$ (where $\Delta_{*}$ denotes the coproduct), Propo- 
sition 4.7 tells us that $J_{*}\left(\beta_{2 n-1}\right) \neq 0$ in $H_{2 n-1}\left(\Omega_{0}^{3} S U(n) ; \mathbb{Z} / 2\right)$. By $(2.4)$, $H_{2 n-1}\left(\Omega_{0}^{3} S U(n) ; \mathbb{Z} / 2\right) \cong \mathbb{Z} / 2$, hence

$$
J_{*}\left(\beta_{2 n-1}\right)= \begin{cases}x_{2} * Q_{1}\left(y_{4 k}\right), & n=4 k+2 \\ x_{2} * z_{8 k+5}, & n=4 k+4\end{cases}
$$

Then using (2.5) we can regard (4.8) as relations in $H_{2 n-1}\left(S U(d) / C_{S U(d)}\right.$; $\mathbb{Z} / 2$ ) for each $d$ for which $\beta_{2 n-1}$ is defined. Therefore (ii) and (iv) of Theorem 2.7 hold. This completes the proof of Theorem 2.7, and hence also that of Theorem A for $G=S U(n)$.

Proof of Theorem 2.8. If $G=S p(1)$, then by the property of the classical $J$-homomorphism (see, for example, [3]), we have

$$
\left\{\begin{array}{l}
J_{*}\left(\alpha_{1}\right)=x_{1} \\
J_{*}\left(\alpha_{2}\right)=y_{2}
\end{array}\right.
$$

Then a similar diagram to (2.5) shows that (4.9) holds for all $n$.

Since $\Delta_{*} J_{*}\left(\alpha_{3}\right)$ contains the term $x_{1} \otimes y_{2}, J_{*}\left(\alpha_{3}\right)$ must contain the term $x_{1} * y_{2}$. (Actually, by [3], we have $J_{*}\left(\alpha_{3}\right)=x_{1} * y_{2}+x_{1}^{3}+Q_{1}\left(x_{1}\right)$.) Similarly, since $\Delta_{*} J_{*}\left(\alpha_{4}\right)$ contains the term $y_{2} \otimes y_{2}$, we can define $z_{4}$ to be $J_{*}\left(\alpha_{4}\right)$.

In general, we can prove the following results.

(i) When $\mu=4 i, \Delta_{*} J_{*}\left(\alpha_{\mu}\right)$ contains the term $J_{*}\left(\alpha_{2 i}\right) \otimes J_{*}\left(\alpha_{2 i}\right)$. Hence we can define $z_{4 i}$ to be $J_{*}\left(\alpha_{4 i}\right)$.

(ii) When $\mu=4 i+1,4 i+2$ or $4 i+3, \Delta_{*} J_{*}\left(\alpha_{\mu}\right)$ contains the term $x_{1} \otimes z_{4 i}$, $y_{2} \otimes z_{4 i}$ or $\left(x_{1} * y_{2}\right) \otimes z_{4 i}$. Hence $J_{*}\left(\alpha_{\mu}\right)$ must contain $x_{1} * z_{4 i}, y_{2} * z_{4 i}$ or $x_{1} * y_{2} * z_{4 i}$.

This completes the proof of Theorem 2.8, and hence also that of Theorem A for $G=S p(n)$.

5. Proof of Theorem 3.4. Let $\nu: S^{n-4} \rightarrow \operatorname{Spin}(n) / C$ be the composition of the following maps:

$$
S^{n-4}=\operatorname{Spin}(n-3) / \operatorname{Spin}(n-4) \stackrel{i}{\rightarrow} \operatorname{Spin}(n) / \operatorname{Spin}(n-4) \stackrel{\pi}{\rightarrow} \operatorname{Spin}(n) / C,
$$

where $i$ is the inclusion and $\pi$ is the projection. First we prove Theorem B(ii). We recall some notations and results about the Clifford algebra from [1].

Let $e_{1}, \ldots, e_{n}$ be a basis of $\mathbb{R}^{n}$ and $C_{n}$ the Clifford algebra. Thus $e_{i}^{2}=$ $-1(1 \leq i \leq n)$ and $e_{i} e_{j}+e_{j} e_{i}=0(i \neq j)$. For $g=e_{i_{1}} \ldots e_{i_{k}} \in C_{n}$, we set $g^{t}=e_{i_{k}} \ldots e_{i_{1}}$.

(1) Let $\operatorname{Spin}(n-4)$ be defined from $\mathbb{R}^{n-4}$ with a basis $e_{1}, \ldots, e_{n-4}$. Then in the principal bundle

$$
\operatorname{Spin}(n-4) \rightarrow \operatorname{Spin}(n-3) \stackrel{p}{\rightarrow} S^{n-4},
$$

the projection $p$ is given by $p(g)=g e_{n-3} g^{t}$. 
(2) Let $e_{i}(n-3 \leq i \leq n)$ be a basis of $\mathbb{R}^{4}$. Recall that $\operatorname{Spin}(4) \cong S_{1}^{3} \times S_{2}^{3}$ (see Section 3 ). We write $S_{i}^{3}$ in terms of the Clifford algebra as follows:

$$
\begin{aligned}
S_{1}^{3}=\left\{\frac{x_{1}}{2}(1\right. & \left.-e_{n-3} e_{n-2} e_{n-1} e_{n}\right)+\frac{x_{2}}{2}\left(e_{n-3} e_{n}+e_{n-2} e_{n-1}\right) \\
& +\frac{x_{3}}{2}\left(-e_{n-3} e_{n-1}+e_{n-2} e_{n}\right)+\frac{x_{4}}{2}\left(e_{n-3} e_{n-2}+e_{n-1} e_{n}\right) \\
& \left.+\frac{1}{2}\left(1+e_{n-3} e_{n-2} e_{n-1} e_{n}\right): \sum_{i=1}^{4} x_{i}^{2}=1\right\}
\end{aligned}
$$

and

$$
\begin{aligned}
S_{2}^{3}=\left\{\frac{x_{1}}{2}(1+\right. & \left.e_{n-3} e_{n-2} e_{n-1} e_{n}\right)+\frac{x_{2}}{2}\left(e_{n-3} e_{n}-e_{n-2} e_{n-1}\right) \\
& +\frac{x_{3}}{2}\left(e_{n-3} e_{n-1}+e_{n-2} e_{n}\right)+\frac{x_{4}}{2}\left(e_{n-3} e_{n-2}-e_{n-1} e_{n}\right) \\
& \left.+\frac{1}{2}\left(1-e_{n-3} e_{n-2} e_{n-1} e_{n}\right): \sum_{i=1}^{4} x_{i}^{2}=1\right\} .
\end{aligned}
$$

As in Section 3 we embed $\operatorname{Spin}(3)$ into $\operatorname{Spin}(n)$ as $S_{1}^{3}$, hence $C=C_{\operatorname{Spin}(n)}=$ $\operatorname{Spin}(n-4) \times S_{2}^{3}$.

We calculate $\operatorname{Ad}(J \circ \nu): S^{n-4} \wedge S^{3} \rightarrow \operatorname{Spin}(n)$ explicitly. Let $u \wedge x \in$ $S^{n-4} \wedge S^{3}$, where $u=\sum_{i=1}^{n-3} u_{i} e_{i}$ with $\sum_{i=1}^{n-3} u_{i}^{2}=1$ and $x \in S_{1}^{3}$. We choose $g \in \operatorname{Spin}(n-3)$ so as to satisfy $g e_{n} g^{t}=u$. (Compare (1).) Then $\operatorname{Ad}(J \circ \nu)(u \wedge x)=g x g^{t} x^{t}$. It is easy to see that

We set

$$
\begin{aligned}
\operatorname{Ad}(J \circ \nu)(u \wedge x)= & \frac{-1+x_{1}}{2} u e_{n-3}+\frac{1+x_{1}}{2} \\
& +\frac{1}{2}\left(-x_{4} e_{n-2}+x_{3} e_{n-1}-x_{2} e_{n}\right)\left(u-e_{n-3}\right) .
\end{aligned}
$$

$$
\operatorname{Pin}^{1}(n)=\operatorname{Pin}(n)-\operatorname{Spin}(n)
$$

We multiply the formula for $\operatorname{Ad}(J \circ \nu)(u \wedge x)$ by $e_{n-3}$ from the right, then we exchange the coefficients of $e_{n-2}$ and $e_{n}$. Thus we obtain a map $\psi$ : $S^{n-4} \wedge S^{3} \rightarrow \operatorname{Pin}^{1}(n)$ defined by

$$
\begin{aligned}
\psi(u \wedge x)= & \frac{1-x_{1}}{2} u+\frac{1+x_{1}}{2} e_{n-3} \\
& +\frac{1}{2}\left(x_{2} e_{n-2}+x_{3} e_{n-1}+x_{4} e_{n}\right)\left(1+u e_{n-3}\right) .
\end{aligned}
$$

In order to prove Theorem $\mathrm{B}(\mathrm{ii})$, it suffices to prove that $\psi$ represents a generator of $\pi_{n-1}\left(\operatorname{Pin}^{1}(n)\right)$.

Recall that for $n \neq 3,7$, the characteristic map $T: S^{n-1} \rightarrow S O(n)$ of the principal bundle $S O(n) \rightarrow S O(n+1) \rightarrow S^{n}$ represents a generator of $\pi_{n-1}(S O(n))$, the first non-stable homotopy group (see, for example, [10]). 
In the Clifford algebra, the generator is given by the inclusion $\iota: S^{n-1} \rightarrow$ $\operatorname{Pin}^{1}(n)$. In Lemma 4.4 for $m=n-4$ and $p=3$, we exchange $u_{1}$ and $u_{n-3}$, and change the order of the coordinates of the right-hand side. Thus we obtain a map $\zeta: S^{n-4} \wedge S^{3} \rightarrow \operatorname{Pin}^{1}(n)$ defined by

$$
\begin{aligned}
\zeta(u \wedge x)= & \frac{1-x_{1}}{2} u+\frac{1+x_{1}}{2} e_{n-3} \\
& +\sqrt{\frac{1-u_{n-3}}{2}}\left(x_{2} e_{n-2}+x_{3} e_{n-1}+x_{4} e_{n}\right) .
\end{aligned}
$$

Now we have two maps, $\psi$ in (5.1) and $\zeta$ in (5.2). Making use of the homotopy

$$
u e_{n-3} \mapsto\left\{t\left(\sum_{i=1}^{n-4} u_{i} e_{i}\right)+u_{n-3} e_{n-3}\right\} e_{n-3},
$$

where $t \in I$, it is easy to construct a homotopy $\psi \simeq \zeta$. This completes the proof of Theorem B(ii).

Now a result which corresponds to Lemma 4.6 and Proposition 4.7 is as follows.

Proposition 5.3. There are choices of the generators $y_{\mu}$ and $z_{\mu}$ such that the following relations hold in $H_{n-4}\left(\Omega_{0}^{3} \operatorname{Spin}(n) ; \mathbb{Z} / 2\right)$ :

(i) $J_{*}\left(\gamma_{n-4}\right)=z_{n-4}$ for $n \equiv 0,2(\bmod 4)$.

(ii) $J_{*}\left(\beta_{n-4}\right)=y_{n-4}$ for $n \equiv 1(\bmod 4)$.

Proof. Let $W$ be the homotopy-theoretic fiber of the inclusion $\Omega_{0}^{3} \operatorname{Spin}(n)$ $\hookrightarrow \Omega_{0}^{3}$ Spin $\simeq B S p$. Consider the following diagram:

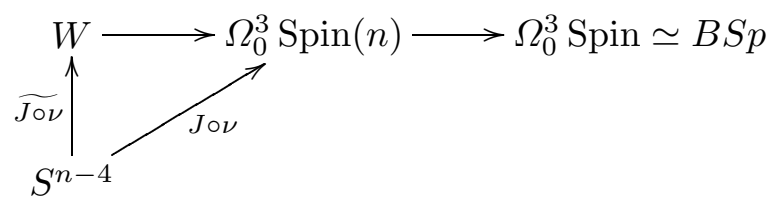

where $\widetilde{J \circ \nu}$ is the lift of $J \circ \nu$. The first non-vanishing homotopy of $W$ is $\pi_{n-4}(W)$ and $\widehat{J \circ \nu}$ is a generator by Theorem $\mathrm{B}(\mathrm{ii})$. If $n \not \equiv 3(\bmod 4)$, then for dimensional reasons, $H_{n-4}(W ; \mathbb{Z} / 2) \rightarrow H_{n-4}\left(\Omega_{0}^{3} \operatorname{Spin}(n) ; \mathbb{Z} / 2\right)$ is injective. Hence $(J \circ \nu)_{*}\left(\sigma_{n-4}\right) \neq 0$, where $\sigma_{n-4}$ is a generator of $H_{n-4}\left(S^{n-4} ; \mathbb{Z} / 2\right)$. By (3.2), we have

$$
\nu_{*}\left(\sigma_{n-4}\right)= \begin{cases}\gamma_{n-4}, & n \equiv 0,2(\bmod 4), \\ \beta_{n-4}, & n \equiv 1(\bmod 4) .\end{cases}
$$

Then we can define $y_{n-4}$ or $z_{n-4}$ to be $J_{*}\left(\gamma_{n-4}\right)$ or $J_{*}\left(\beta_{n-4}\right)$. (More precisely, $J_{*}\left(\gamma_{n-4}\right)$ for $n \equiv 6(\bmod 8)$ and $J_{*}\left(\beta_{n-4}\right)$ for $n \equiv 5(\bmod 8)$ must be the right-hand side of $(3.3)$, since $H_{n-4}\left(\Omega_{0}^{3} \operatorname{Spin}(n) ; \mathbb{Z} / 2\right) \cong \mathbb{Z} / 2$.) 
Proof of Theorem 3.4. First, Theorem 3.4(i) follows from the homotopy commutative diagram

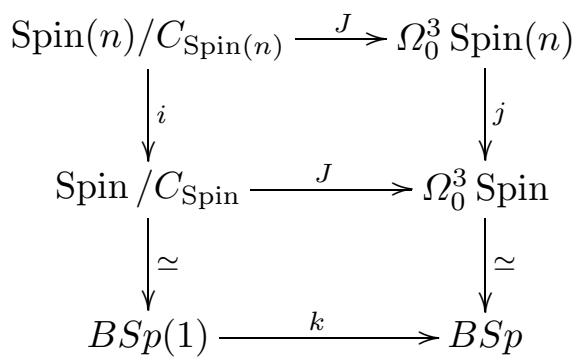

where $i, j$ and $k$ are the inclusions. (The homotopy commutativity of the bottom square is proved in the same way as in the third diagram of $[9, \mathrm{p}$. 4054] for $k=1$ and $l=\infty$.)

Next, (ii) and (iii) of Theorem 3.4 are proved using Proposition 5.3 and a diagram similar to (2.5). (Compare the proof of Theorem 2.7 in Section 4.) This completes the proof of Theorem 3.4, and hence also that of Theorem A for $G=\operatorname{Spin}(n)$.

REMARK. Using Theorem 3.4, we can obtain information on $J_{*}(\alpha)$ for each generator $\alpha$ of $H_{*}(\operatorname{Spin}(n) / C ; \mathbb{Z} / 2)$. For example, consider an element of the form $\alpha=\left(u_{i_{1}}^{\varepsilon_{1}} u_{i_{2}}^{\varepsilon_{2}} u_{i_{3}}^{\varepsilon_{3}}\right)^{*}$, where $\varepsilon_{j}=0$ or 1. (For the generators $u_{i_{j}}$, see Proposition 3.1, and for the notation $(\cdot)^{*}$, see (3.2).) We write $\xi_{\mu}$ for $y_{\mu}$ or $z_{\mu}$. Then we can prove that $J_{*}(\alpha)$ contains the term $\xi_{i_{1}}^{\varepsilon_{1}} * \xi_{i_{2}}^{\varepsilon_{2}} * \xi_{i_{3}}^{\varepsilon_{3}}$. For the proof, consider the coproduct $\Delta_{*}$.

\section{References}

[1] M. F. Atiyah, R. Bott and A. Shapiro, Clifford modules, Topology 3 (suppl. 1) (1964), 3-38.

[2] R. Bott, The space of loops on a Lie group, Michigan Math. J. 5 (1958), 35-61.

[3] C. P. Boyer, B. M. Mann and D. Waggoner, On the homology of $S U(n)$ instantons, Trans. Amer. Math. Soc. 323 (1991), 529-561.

[4] Y. Choi, Homology of the double and triple loop space of $S O(n)$, Math. Z. 222 (1996), 59-80.

[5] - The mod 2 homology of $S p(n)$ instantons and the classifying space of the gauge group, Bull. Austral. Math. Soc. 55 (1997), 503-512.

[6] Y. Choi and Y. Seonhee, Homology of the double and triple loop spaces of $E_{6}, E_{7}$, and $E_{8}$, Manuscripta Math. 103 (2000), 101-116.

[7] Y. Kamiyama, A. Kono and M. Tezuka, Cohomology of the moduli space of $S O(n)$ instantons with instanton number 1 , preprint.

[8] M. Mimura and H. Toda, Topology of Lie Groups. I, II, Transl. Math. Monographs 91, Amer. Math. Soc., Providence, RI, 1991.

[9] M. Sanders, Classifying spaces and Dirac operators coupled to instantons, Trans. Amer. Math. Soc. 347 (1995), 4037-4072. 
[10] N. Steenrod, The Topology of Fibre Bundles, Princeton Math. Ser. 14, Princeton Univ. Press, Princeton, 1951.

[11] G. W. Whitehead, Elements of Homotopy Theory, Grad. Texts in Math. 61, Springer, 1978.

Department of Mathematics

University of the Ryukyus

Okinawa 903-0213, Japan

E-mail: kamiyama@sci.u-ryukyu.ac.jp

Received 20 January 2003 